\title{
A sliding mode control for a wound rotor synchronous generator with an isolated RL load
}

\author{
R.S. Muñoz-Aguilar, A. Dòria-Cerezo and E. Fossas
}

\begin{abstract}
In this paper a sliding mode controller for the stator voltage amplitude of a stand-alone wound rotor synchronous generator is presented. The standard dq-model of the electrical machine connected to an isolated inductive load is obtained. Then, a control law is designed in the framework of sliding mode control. The controller introduces a dynamic extension so that the stator amplitude relative degree is one. Numerical simulations are also presented to validate the presented control law.
\end{abstract}

\section{INTRODUCTION}

The wound rotor synchronous machine (WRSM) is a doubly-fed electrical machine which can be used for both: generation [2][3] and driving applications [1][8]. Usually, wound rotor synchronous generators are studied when they are directly connected to the power grid [2]. In this case, the stator voltage and frequency are set by the power grid, while the rotor voltage helps to improve the power factor and to compensate the reactive power at the connection point. In this paper we study the isolated load case which is significantly different: neither amplitude nor frequency of stator voltage are fixed. For the stand-alone configuration, although the mechanical speed determines the frequency, the rotor voltage is used to set the stator amplitude.

The WRSM is controlled by several techniques, in industry the most common are linear techniques [5][9], however, decoupling methods [4], which widely employed for asynchronous machines, are also extended in the synchronous case. Modern non-linear techniques such as passivity-based control [1] or predictive control [7], are also used for regulating this kind of machine.

Sliding Mode Control (SMC) has been proposed as an appropriate technique for controlling electrical machines [10]. For instance, the classical approach of the SMC was used for a position servo system [6]. More recently, sliding observers combined with control linearization and singular perturbations, are applied to a synchronous generator connected to a power grid [2]. Higher order sliding modes, have also been proposed in [11] for avoiding chattering in a wind energy generator module power control.

The main goal of this work is to design a sliding mode control algorithm for a wound rotor synchronous generator feeding an isolated resistive-inductive unknown load.

R.S. Muñoz-Aguilar and A. Dòria-Cerezo are with the Department of Electrical Engineering and the Institute of Industrial and Control Engineering, Universitat Politècnica de Catalunya, Spain \{raul.munoz-aguilar, arnau.doria\}@upc.edu

E. Fossas is with the Institute of Industrial and Control Engineering, Universitat Politècnica de Catalunya, 08028 Barcelona, Spain enric.fossaslupc.edu
In this case, sliding mode control cannot be directly applied to the system natural output because it is relative degree zero. Instead, a dynamical extension of the system is proposed so that the output relative degree is one.

The paper is organized as follows. In Section II the wound rotor synchronous machine model is presented and the control goals are described. Section III concerns the Sliding Mode controller design, while simulation results are shown in Section IV. Finally, conclusions are drawn in Section V.

\section{SYSTEM DESCRIPTION}

In this Section the dynamical model of the system is presented. Figure 1 shows the following scenario: an Internal Combustion Engine (ICE) drags a WRSM, which acts as a generator to feed an isolated load.

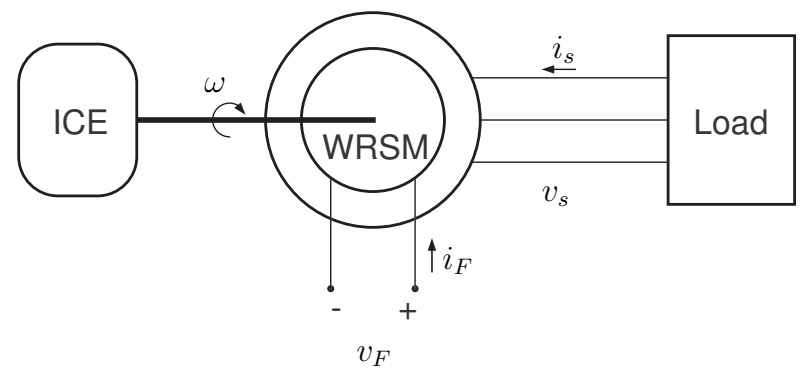

Fig. 1. Scheme of an isolated wound rotor synchronous machine with an isolated load.

As explained before, this system differs from the typical grid connection, in which, the frequency and the voltage amplitude are set by the grid. In an isolated connection the frequency is determined by the mechanical speed (provided by the ICE), while the voltage amplitude must be assured by the rotor field voltage.

\section{A. Dynamical model}

From the well-known WRSM dynamical equations (in the dq coordinates), and the interconnection rules with an inductive load, the whole dynamic system is presented.

Assuming that the mechanical speed is externally regulated by the ICE, the electrical part of the wound rotor synchronous machine can be described as

$$
L \frac{\mathrm{d} x}{\mathrm{~d} t}=\left(\begin{array}{ccc}
-R_{s} & \omega L_{s} & 0 \\
-\omega L_{s} & -R_{s} & -\omega L_{m} \\
0 & 0 & -R_{F}
\end{array}\right) x+\left(\begin{array}{c}
v_{d} \\
v_{q} \\
v_{F}
\end{array}\right)
$$


where

$$
L=\left(\begin{array}{ccc}
L_{s} & 0 & L_{m} \\
0 & L_{s} & 0 \\
L_{m} & 0 & L_{F}
\end{array}\right)
$$

is the inductance matrix, $x^{T}=\left(i_{d}, i_{q}, i_{F}\right) \in \mathbb{R}^{3}$ are the dqstator and field currents, $R_{s}$ and $R_{F}$ are the stator and field resistances, $L_{s}, L_{m}$ and $L_{F}$ are the stator, mutual and field inductances, $\omega$ is the mechanical speed ${ }^{1}, v_{d}, v_{q}$ and $v_{F}$ are the dq-stator voltages and the field voltage which will be used as a control input.

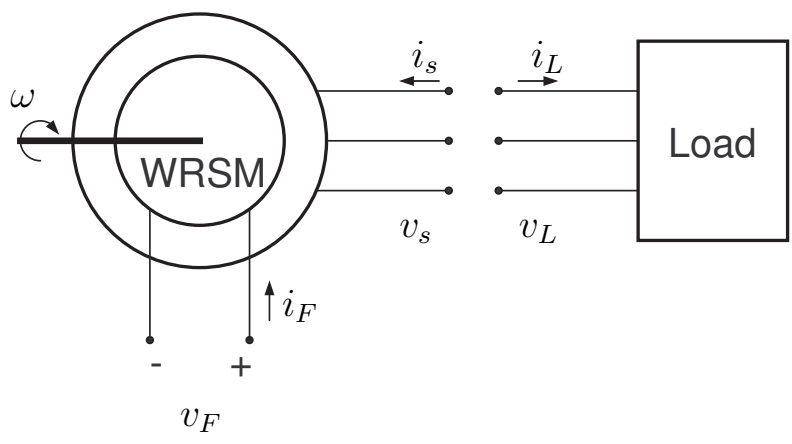

Fig. 2. Detail of the interconnection between a wound rotor synchronous machine and an inductive load.

In order to design the control law, let us obtain the complete model of a WRSM connected to an inductive load, which is modeled as a pure resistive element, $R_{L}$, in series with a pure inductive element, $L_{L}$. The interconnection scheme is depicted in Figure 2, where $v_{L}^{T}=\left(v_{L d}, v_{L q}\right) \in \mathbb{R}^{2}$ and $i_{L}^{T}=\left(i_{L d}, i_{L q}\right) \in \mathbb{R}^{2}$ are the dq load voltages and currents, which are related by

$$
v_{L}=\left(R_{L} I_{2}+\omega L_{L} J_{2}\right) i_{L}+L_{L} \frac{\mathrm{d}}{\mathrm{d} t} i_{L}
$$

where

$$
I_{2}=\left(\begin{array}{ll}
1 & 0 \\
0 & 1
\end{array}\right) \quad J_{2}=\left(\begin{array}{cc}
0 & -1 \\
1 & 0
\end{array}\right) .
$$

The interconnection rules, according to Fig. 2, are

$$
\begin{aligned}
& v_{s}=v_{L} \\
& i_{L}=-i_{s} .
\end{aligned}
$$

Now, putting together (1), (2) and (3), the system can be written in an affine form as

$$
\hat{L} \frac{\mathrm{d} x}{\mathrm{~d} t}=A x+G v_{F},
$$

where $\hat{L}$ is a new inductance matrix,

$$
\hat{L}=\left(\begin{array}{ccc}
L_{s}+L_{L} & 0 & L_{m} \\
0 & L_{s}+L_{L} & 0 \\
L_{m} & 0 & L_{F}
\end{array}\right)
$$

\footnotetext{
${ }^{1}$ For simplicity a two-poles machine is considered.
}

and the homogeneous dynamics $A$ and the input vector $G$ are given by

$$
A=\left(\begin{array}{ccc}
-\left(R_{s}+R_{L}\right) & \omega\left(L_{s}+L_{L}\right) & 0 \\
-\omega\left(L_{s}+L_{L}\right) & -\left(R_{s}+R_{L}\right) & -\omega L_{m} \\
0 & 0 & -R_{F}
\end{array}\right),
$$

and

$$
G=\left(\begin{array}{l}
0 \\
0 \\
1
\end{array}\right)
$$

\section{B. Control objective}

As mentioned before, this must ensure stator voltage amplitude and frequency. The synchronous machine stator frequency is directly given by the mechanical speed, which, in this paper is assumed to be constant and externally regulated. Then, the system output is the stator voltage amplitude $V_{s}$, which can be obtained, in a dq-framework as

$$
V_{s}=\sqrt{v_{d}^{2}+v_{q}^{2}},
$$

where $v_{d}$ and $v_{q}$ are defined by (2) and (3), and the current derivative in (2) can be obtained from (4). This leads to

$$
\begin{aligned}
\mu_{d} \frac{d i_{d}}{d t}= & L_{F}\left(-\left(R_{s}+R_{L}\right) i_{d}+\omega\left(L_{s}+L_{L}\right) i_{q}\right) \\
& -L_{m}\left(-R_{F} i_{F}+v_{F}\right) \\
\mu_{q} \frac{d i_{q}}{d t}= & -\omega\left(L_{s}+L_{L}\right) i_{d}-\left(R_{s}+R_{L}\right) i_{q} \\
& -\omega L_{m} i_{F} .
\end{aligned}
$$

where $\mu_{d}=L_{F}\left(L_{s}+L_{L}\right)-L_{m}^{2}$ and $\mu_{q}=L_{s}+L_{L}$. Notice that, while the system dynamics is linear, as equation (4) states, the desired output, $V_{s}$, is a highly tedious nonlinear function, which can be obtained replacing (6) and (7) in (2), and then in (5). As explained before, the control input is the field voltage $v_{F}$.

Notice that for a pure resistive load the stator voltage is relative degree one in relation to $v_{F}$ [3]. However, for isolated loads, including inductive terms, the stator voltage is relative degree zero. It is appropriate to mention here that to have relative degree one is a necessary condition for a switching surface to have sliding modes on it. Hence, the standard procedure used in the resistive case has to be modified.

\section{CONTROL DESIGN}

This section deals with the design of a sliding mode controller for an isolated wound rotor synchronous machine feeding an inductive load. As mentioned before, a system dynamics extension is carried out in order to have a relative degree one sliding surface. The ideal sliding dynamics is analyzed by means of the equivalent control.

\section{A. Sliding surface and equivalent control}

According to the control goals we define sliding surface $s(x)$ as

$$
s(x)=V_{s}^{2}-V_{r e f}^{2},
$$


where $V_{\text {ref }}$ is the stator voltage amplitude reference. Using (2), (5) and tacking into account (3), sliding surface can be given as a function of the state variables

$$
\begin{aligned}
s(x)= & \left(-R_{L} i_{d}+\omega L_{L} i_{q}-L_{L} \frac{\mathrm{d} i_{d}}{\mathrm{~d} t}\right)^{2} \\
& +\left(-R_{L} i_{q}-\omega L_{L} i_{d}-L_{L} \frac{\mathrm{d} i_{q}}{\mathrm{~d} t}\right)^{2}-V_{r e f}^{2}
\end{aligned}
$$

where the expressions for $\frac{\mathrm{d} i_{d}}{\mathrm{~d} t}$ and $\frac{\mathrm{d} i_{q}}{\mathrm{~d} t}$ are respectively given in (6) and (7).

As $\frac{\mathrm{d} i_{d}}{\mathrm{~d} t}$ depends on $v_{F}, s(x)$ is relative degree zero. Thus, a system dynamic extension is defined through equation (4) and

$$
\frac{\mathrm{d} v_{F}}{\mathrm{~d} t}=k u,
$$

where $\mathrm{k}$ is a positive constant and the new state and input variables respectively are $z^{T}=\left(i_{d}, i_{q}, i_{F}, v_{F}\right)$ and $\mathrm{u}$.

Let us to rewrite the extended system, as

$$
\mathcal{L} \frac{\mathrm{d} z}{\mathrm{~d} t}=\mathcal{A} z+\mathcal{G} u
$$

where

$$
\begin{gathered}
\mathcal{A}=\left(\begin{array}{cccc}
-\left(R_{s}+R_{L}\right) & \omega\left(L_{s}+L_{L}\right) & 0 & 0 \\
-\omega\left(L_{s}+L_{L}\right) & -\left(R_{s}+R_{L}\right) & -\omega L_{m} & 0 \\
0 & 0 & -R_{F} & 1 \\
0 & 0 & 0 & 0
\end{array}\right) \\
\mathcal{L}=\left(\begin{array}{cc}
\hat{L} & 0 \\
0 & 1
\end{array}\right)
\end{gathered}
$$

and

$$
\mathcal{G}=\left(\begin{array}{l}
0 \\
0 \\
0 \\
k
\end{array}\right)
$$

The equivalent control, $u_{e q}$, is defined so that $\dot{s}=0$,

$$
\frac{\mathrm{d} s}{\mathrm{~d} t}=\frac{\partial s}{\partial z} \frac{\mathrm{d} z}{\mathrm{~d} t}=0 .
$$

Using (11) this is equivalent to ,

$$
\frac{\partial s}{\partial z} \mathcal{L}^{-1}\left(\mathcal{A} z+\mathcal{G} u_{e q}\right)=0 .
$$

Hence

$$
u_{e q}=-\left(\frac{\partial s}{\partial z} \mathcal{L}^{-1} \mathcal{G}\right)^{-1} \frac{\partial s}{\partial z} \mathcal{L}^{-1} \mathcal{A} z
$$

From (8),

$$
\frac{\partial s}{\partial z}=\frac{\partial v_{d}^{2}}{\partial z}+\frac{\partial v_{q}^{2}}{\partial z}=2\left(v_{d} \frac{\partial v_{d}}{\partial z}+v_{q} \frac{\partial v_{q}}{\partial z}\right)
$$

Since $\mathcal{G}$ has zeros in the first, second and third row, $\mathcal{L}^{-1}$ contains only a nonzero term in the fourth row and $v_{q}$ does not depends on $v_{F}$, we obtain

$$
\begin{aligned}
\frac{\partial s}{\partial z} \mathcal{L}^{-1} \mathcal{G} & =v_{d} \frac{\partial}{\partial v_{F}}\left(L_{L} \frac{\mathrm{d} i_{d}}{\mathrm{~d} t}\right) k \\
& =2 k \frac{L_{L} L_{m}}{\mu_{d}} v_{d}
\end{aligned}
$$

Hence,

$$
u_{e q}=-\frac{\mu_{d}}{2 k L_{L} L_{m}} \frac{1}{v_{d}}\left(\frac{\partial s}{\partial z} \mathcal{L}^{-1} \mathcal{A} z\right) .
$$

Note that the sliding motion can only be expected in the subspace defined by $v_{d} \neq 0$. This result was already obtained for the resistive case [3], where $v_{d}=-R_{L} i_{d}$, and

$$
u_{e q}=-\frac{\left(L_{s} L_{F}-L_{m}^{2}\right)}{2 L_{m} R_{L}^{2}} \frac{1}{i_{d}}\left(\frac{\partial s}{\partial x} L^{-1} A x\right) .
$$

\section{B. Equilibrium points}

Equilibrium points of (11) are also a solution of (4)

$$
x^{*}\left(v_{F}\right)=A^{-1} G v_{F}
$$

which can be computed as

$$
x^{*}\left(v_{F}\right)=\left[\begin{array}{c}
-\frac{\omega^{2}\left(L_{s}+L_{L}\right) L_{m}}{R_{F}\left(\left(R_{s}+R_{L}\right)^{2}+\omega^{2}\left(L_{s}+L_{L}\right)^{2}\right)} \\
-\frac{\omega\left(R_{s}+R_{L}\right) L_{m}}{R_{F}\left(\left(R_{s}+R_{L}\right)^{2}+\omega^{2}\left(L_{s}+L_{L}\right)^{2}\right)} \\
\frac{1}{R_{F}}
\end{array}\right] v_{F} .
$$

To obtain $v_{F}^{*}$, (15) can be replaced into (9), yielding

$$
v_{F}^{*}= \pm \frac{\sqrt{D} R_{F} V_{r e f}}{\left(\omega^{2} L_{L}^{2}+R_{L}^{2}\right) \omega L_{m}}
$$

where $D=\left(\omega^{2} L_{L}^{2}+R_{L}^{2}\right)\left(\left(R_{s}+R_{L}\right)^{2}+\omega^{2}\left(L_{s}+L_{L}\right)^{2}\right)$. Finally (16) can be used in (15) to obtain $i_{d}^{*}, i_{q}^{*}$ and $i_{F}^{*}$.

\section{Ideal Sliding Dynamics}

The Ideal Sliding Dynamics (ISD) is defined on the sliding surface presumed that it is invariant by the flow. From (11), replacing $u=u_{e q}$ together with $s(x)=0$, the ISD could be rewritten as

$$
\dot{x}=\hat{L}^{-1}\left(A x+G v_{F}(x)\right),
$$

where now $v_{F}(x)$ comes from solving (9) for $v_{F}$.

Note also that the extended system is symmetric with respect to the origin. It is clear for the linear part of the dynamics defined by matrix $A$, while for the nonlinear part of $v_{F}(x)$ notice that $s\left(x, v_{F}\right)=s\left(-x,-v_{F}\right)$. Consequently, it is sufficient to analyze stability in one of the two equilibria given by (15) and (16).

As a first approximation, since this system is highly nonlinear, the stability of the small-signal model around the equilibrium point will be analysed. Linearizing the ISD, (17), around the equilibrium point, we obtain

$$
\begin{aligned}
\hat{L} \dot{x} \simeq & \left(\left.\frac{\partial}{\partial x}\left(A x+G v_{F}(x)\right)\right|_{x=x^{*}}\right)\left(x-x^{*}\right) \\
& +A x^{*}+G v_{F}\left(x^{*}\right) .
\end{aligned}
$$

In this case the stability is given by the eigenvalues of

or

$$
\left.\hat{L}^{-1} \frac{\partial}{\partial x}\left(A x+G v_{F}(x)\right)\right|_{x=x^{*}}
$$

$$
\hat{L}^{-1}\left(A+\left.G \frac{\partial v_{F}}{\partial x}\right|_{x=x^{*}}\right),
$$

that have to lie in the left half plane. This analysis involves a tedious equation, but as a first approximation the stability 
can be studied numerically. In simulation results, in Section IV, numerical computations have been done to determine the eigenvalues of (18).

\section{Sliding Mode Controller}

Sliding mode controller is in charge of approaching trajectories to the sliding surface and, when it is reached, the controller forces trajectories to remain on the surface (sliding modes). In order to define the control action for the extended system (11) and the sliding surface given in (8), let us consider the Lyapunov function candidate,

$$
V=\frac{1}{2} s^{2}
$$

The discontinuous control action ought to guarantee $s \dot{s} \leq 0$, which is equivalent to,

$$
s \frac{\partial s}{\partial z} \mathcal{L}^{-1}(\mathcal{A} z+\mathcal{G} u)<0 .
$$

Adding and substracting $\mathcal{G} u_{e q}$ and taking into account (12), this equation is equivalent to

$$
s \frac{\partial s}{\partial z} \mathcal{L}^{-1} \mathcal{G}\left(u-u_{e q}\right)<0 .
$$

Hence, the control action

$$
u=u_{e q}-\operatorname{sign}\left(s \frac{\partial s}{\partial z} \mathcal{L}^{-1} \mathcal{G}\right)
$$

yields

$$
\dot{V}=-\left|s \frac{\partial s}{\partial z} \mathcal{L}^{-1} \mathcal{G}\right| \leq 0 .
$$

If the control input $u$ takes values in the discrete set $\left\{u_{1}, u_{2}\right\}$, where $u_{1}<u_{2}$, the control law can be defined as

$$
u=\left\{\begin{array}{ll}
u_{1} & \text { if } \quad s \frac{\partial s}{\partial z} \mathcal{L}^{-1} \mathcal{G}>0 \\
u_{2} & \text { if } \quad s \frac{\partial s}{\partial z} \mathcal{L}^{-1} \mathcal{G} \leq 0
\end{array},\right.
$$

which also assures that $\dot{V} \leq 0$ in the in the sliding surface subset defined by $\min \left\{u_{1}, u_{2}\right\} \leq u_{e q} \leq \max \left\{u_{1}, u_{2}\right\}$. Using (13), and taking into account that $\frac{2 k L_{L} \bar{L}_{m}}{\mu_{d}}>0$, the switching policy

$$
u= \begin{cases}u_{1} & \text { if } \quad-s v_{d}>0 \\ u_{2} & \text { if } \quad-s v_{d} \leq 0\end{cases}
$$

ensures $s \dot{s} \leq 0$ presumed that the equivalent control fulfills the later inequalities. Note that the (fictitious) control law, $u$, is a simple function which does not depend on the load values, neither on the machine parameters. Only $v_{d}$ and $v_{q}$ (which are measurable outputs) are required.

A more complicated task is to determine, analytically, the range of $\left[u_{1}, u_{2}\right]$ to ensure sliding. This is not trivial because $u_{e q}$ is a complex function and it depends on the state values, see equation (14). A simple analysis shows that in the equilibrium $u_{e q}^{*}=0$. Actually, effectively, from (14),

$$
u_{e q}^{*}=-\frac{\mu_{d}}{2 k L_{L} L_{m}} \frac{1}{v_{d}^{*}}\left(\frac{\partial s}{\partial z} \mathcal{L}^{-1} \mathcal{A} z^{*}\right),
$$

thus, replacing it on (11) yields $\mathcal{A} z^{*}=0$. Hence, the only condition to ensure sliding close to equilibria of the ideal sliding dynamics is $u_{1}<0<u_{2}$.
The rotor voltage can be obtained from (10)

$$
v_{F}=k \int u \mathrm{~d} t .
$$

In a real application the actual control input, $v_{F}$ has to lie in a given bounded set. Note that this should not necessarily constrain the values the fictitious variables $u_{1}$ and $u_{2}$ take.

Figure 3 shows the proposed control scheme. Note that, as a result of the control design, the error of the square of the stator voltage amplitude is multiplied by the $v_{d}$ voltage. The resulting control action, $v_{F}$ is a continuous signal which would be implemented using a PWM.

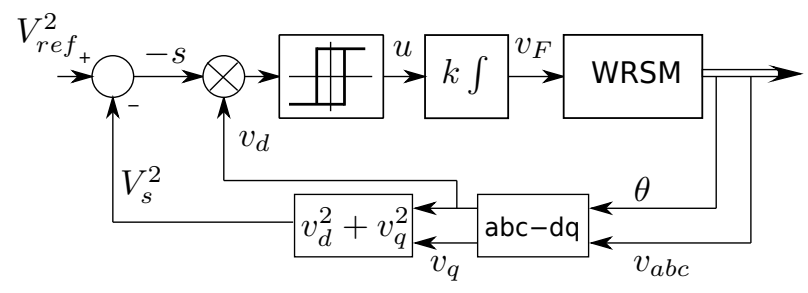

Fig. 3. Control scheme

\section{Simulations}

In this section the simulations results using the designed controller are presented. The WRSM is a $40 \mathrm{~kW}$ machine with the following parameters: $L_{s}=26.25 \mathrm{mH}, R_{s}=$ $0.181 \Omega, L_{m}=25.29 \mathrm{mH}, L_{F}=27.19 \mathrm{mH}, R_{F}=0.1002 \Omega$ and the mechanical speed is fixed at $\omega=314 \mathrm{rad} \mathrm{s}^{-1}$. Initial conditions holds $V_{s}=400 \mathrm{~V}$ and the resistive-inductive load is defined trough the values $R_{L}=4 \Omega$ and $L_{L}=10 \mathrm{mH}$. As for the control action, $k=1, u_{1}=-10^{5}$ and $u_{2}=10^{5}$ and it is implemented by means of an hysteresis function.

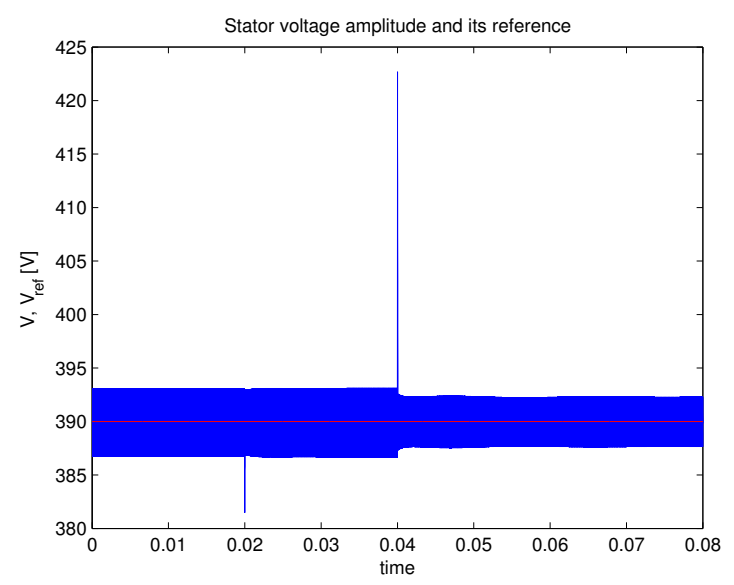

Fig. 4. Simulation results: stator voltage amplitude, $V_{s}$.

Two numerical experiments are run in order to evaluate the closed loop system performance: the load is perturbed in both, the resistive and inductive components, and some steps are considered in the reference. In the first test, load resistance $R_{L}$ varies from $4 \Omega$ to $3.7 \Omega$ at $t=20 \mathrm{~ms}$ then, at $t=40 \mathrm{~ms}$, load inductance changes from $L_{L}=10 \mathrm{mH}$ to $L_{L}=5 \mathrm{mH}$. 
The stator voltage amplitude, $V_{s}$, is depicted in Fig. 4. Note that, even $R_{L}, L_{L}$ values are suddenly modified, the steady state value of the output is the desired one. Additionally, the convergence-time is small.

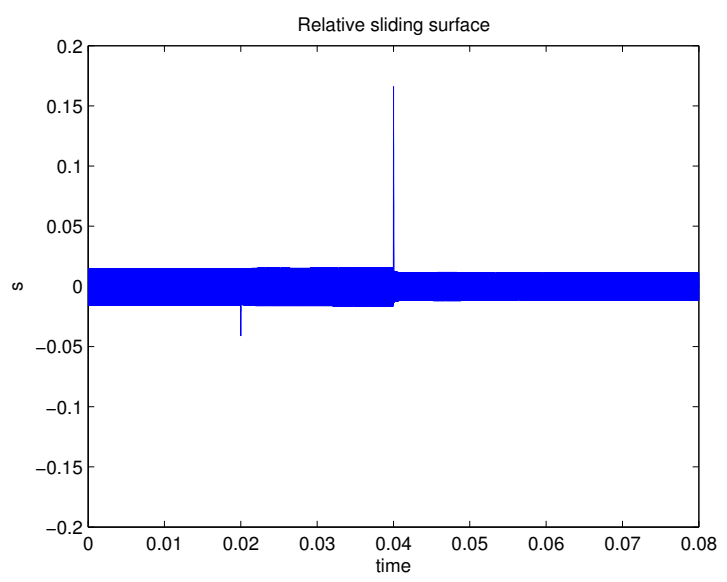

Fig. 5. Simulation results: sliding surface, $s$.

The sliding surface, $s$, is shown in Fig. 5. The selected values of $u_{1}$ and $u_{2}$ allow to keep the system on the sliding surface, which chatters around zero.

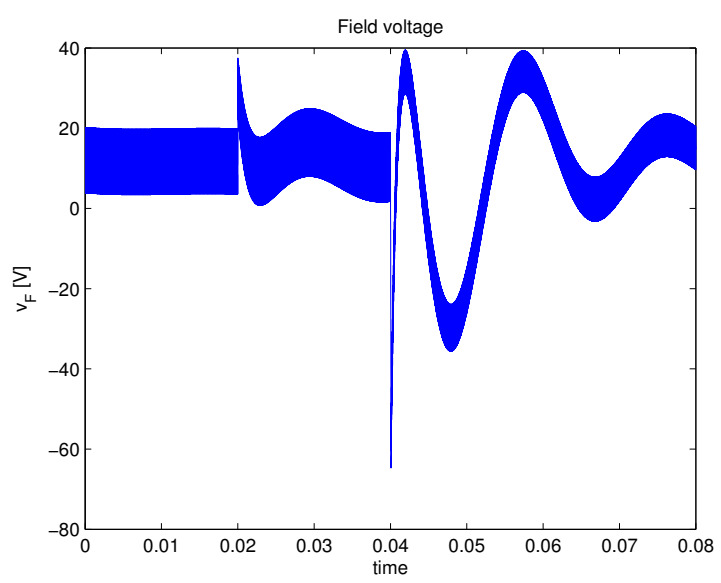

Fig. 6. Simulation results: field voltage and equivalent control actions, $v_{F}$, $u_{e q}$.

Fig. 6 shows the field voltage, $v_{F}$ which remains in real implementable values. Note the dynamics of the control action is slower than the output dynamics. This is the effect of the ISD. Fig. 7 shows the three-phase stator voltages and currents, $v_{a b c}, i_{a b c}$ and field current $i_{F}$, respectively.

In the second experiment the reference of the stator voltage amplitude is modified asFig. 8 shows. The same figure shows that the desired stator voltage amplitude is reached in a short time.

Fig. 9 shows the sliding surface behavior which keeps near zero.

The field voltage, $v_{F}$ remains in the expected boundary as Fig. 10 shows. The three-phase stator voltage and currents, $v_{a b c}, i_{a b c}$, and field current, $i_{F}$, respectively can be appreciated in Fig. 11.
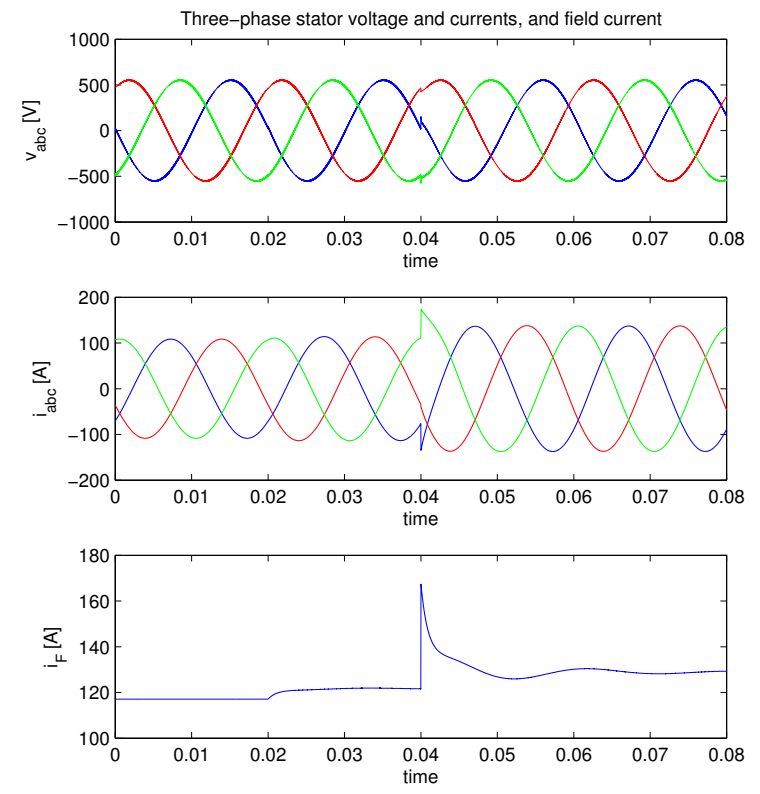

Fig. 7. Simulation results: three-phase stator voltage and currents and field current, $v_{a b c}, i_{a b c}$ and $i_{F}$.

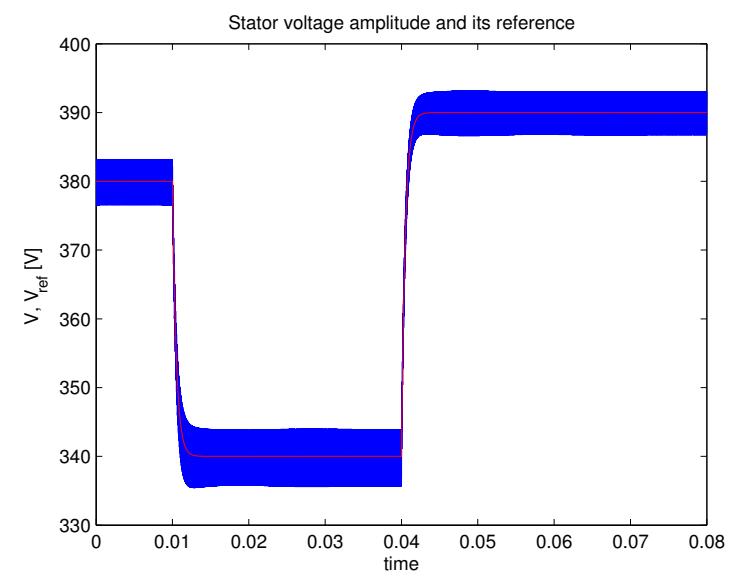

Fig. 8. Simulation results: stator voltage amplitude, $V_{s}$.

\section{CONCLUSIONS}

In this paper a Sliding Mode controller has been designed for a WRSM acting as a generator for an isolated resistiveinductive load. As result, a robust closed loop performance, which neither depends on the machine parameters nor on the load values, is obtained.

The stability of the closed loop system has also been numerically checked. Although it shows a rich nonlinear behaviour, the two equilibrium points are locally asymptotically stable.

Further research includes the study of nonlinear loads and the experimental validation of the controller in a real plant.

\section{ACKNOWLEDGMents}

R. S. Muñoz-Aguilar and A. Dòria-Cerezo were partially supported by the Spanish government research projects ENE2009-13998-C02-01 and DPI2007-62582, respectively. 


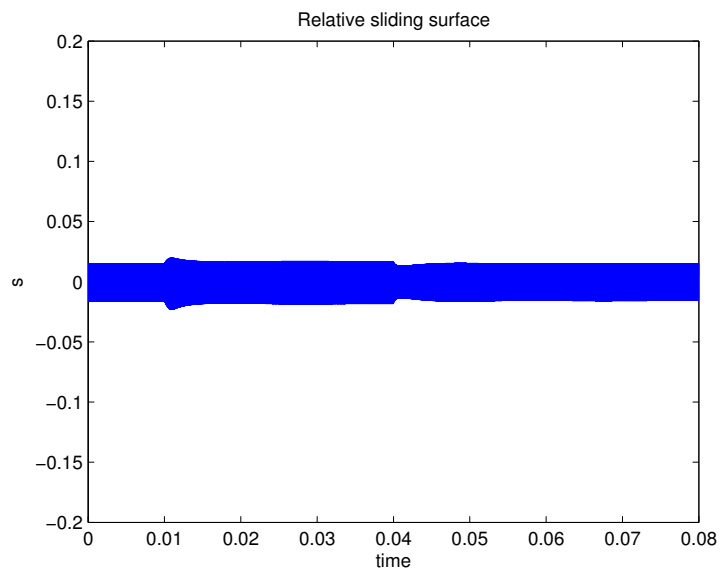

Fig. 9. Simulation results: sliding surface, $s$.

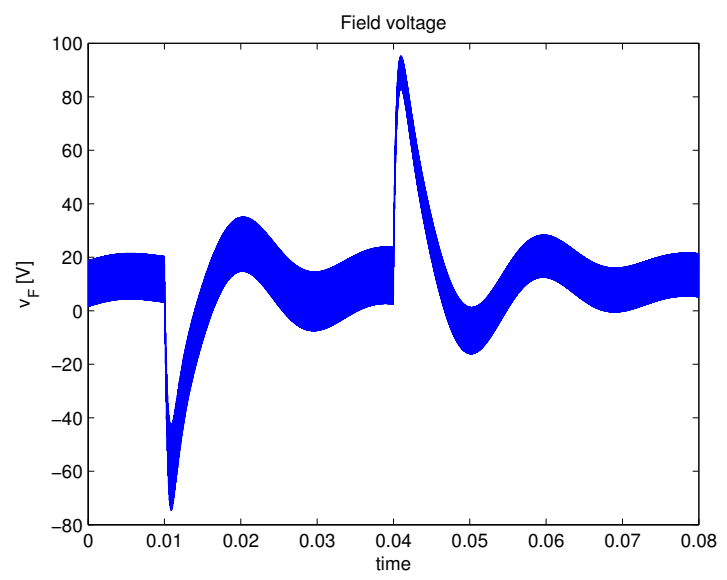

Fig. 10. Simulation results: field voltage control action, $v_{F}$.

E. Fossas was also partially supported by the Spanish government research projects DPI2007-62582 and DPI2008-01408.

\section{REFERENCES}

[1] C. Batlle, A. Dòria-Cerezo, and G. Espinosa. Simultaneous idapassivity-based control of a wound rotor synchronous motor. In Proc. IEEE Conf. on Decision and Control, 2008.

[2] J. Cabrera-Vázquez, A.G. Loukianov, J.M. Cañedo, and V.I. Utkin. Robust controller for synchronous generator with local load via vsc. Electrical Power and Energy Systems, 29:348-359, 2007.
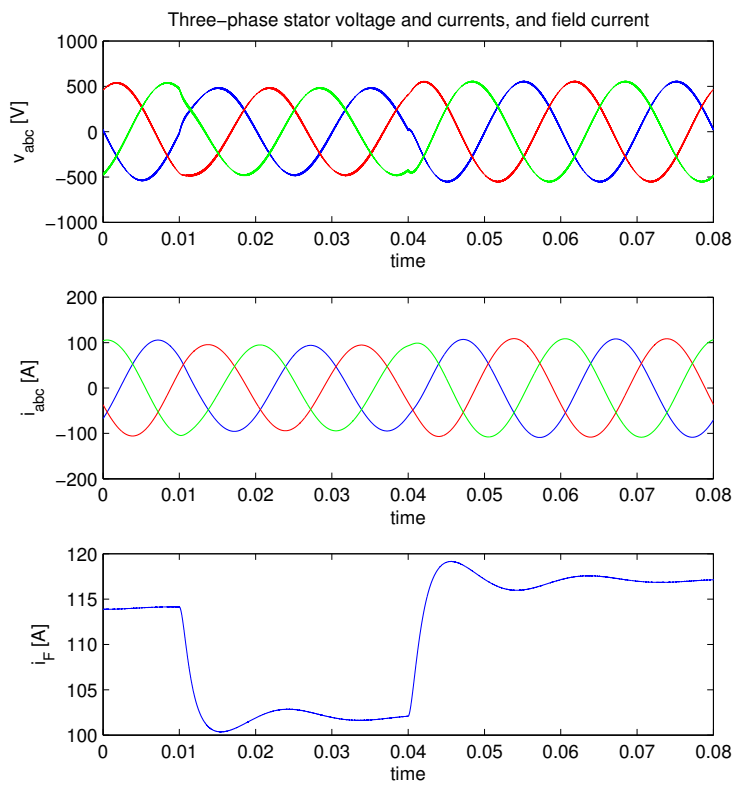

Fig. 11. Simulation results: three-phase stator voltage and currents and field current, $v_{a b c}, i_{a b c}$ and $i_{F}$.

[3] A. Dòria-Cerezo, E. Fossas, R.S. Muñoz-Aguilar, and V.I. Utkin. Sliding mode control of an isolated wound rotor synchronous generator. In Proc. European Control Conference, 2009.

[4] E. Ho and P.C. Sen. High-performance decoupling control techniques for various rotating field machines. IEEE Trans. on Industrial Electronics, 42(1):40-49, 1995.

[5] W. Leonhard. Control of electric drives. Springer, 1995.

[6] C. Namuduri and P.C. Sen. A servo-control system using a selfcontrolled synchronous motor ( $\mathrm{scsm}$ ) with sliding mode controller. IEEE Trans. on Industry Applications, 23(2):283-295, 1987.

[7] M-W. Naouar, E. Monmasson, A. Naassani, I. Slama-Belkhodja, and N. Patin. Fpga-based current controllers for ac machine drives-a review. IEEE Trans. on Industrial Electronics, 54(4):1907-1925, 2007.

[8] C. Rossi, D. Casadei, A. Pilati, and M. Marano. Wound rotor salient pole synchronous machine drive for electric traction. In Proc. IEEE Industry Applications Conference, 2006.

[9] M.K. Senesky, P. Tsao, and S.R. Sanders. Simplified modelling and control of a synchronous machine with variable-speed six-step drive. In Proc. IEEE Applied Power Electronics Conference and Exposition, 2004.

[10] V. Utkin, J. Guldner, and J. Shi. Sliding Mode Control in Electromechanical Systems. Taylor and Francis, 1999.

[11] F. Valenciaga and P.F. Puleston. High-order sliding control for a wind energy conversion system based on a permanent magnet synchronous generator. IEEE Trans. on Energy Conversion, 23(3), 2008. 